\title{
A Naïve Sticky Information Model of Households' Inflation Expectations
}

\author{
Markku Lanne \\ University of Helsinki, RUESG and HECER \\ and \\ Arto Luoma \\ University of Tampere \\ and \\ Jani Luoto \\ University of Jyväskylä
}

Discussion Paper No. 216

April 2008

ISSN 1795-0562

HECER - Helsinki Center of Economic Research, P.O. Box 17 (Arkadiankatu 7), FI-00014 University of Helsinki, FINLAND, Tel +358-9-191-28780, Fax +358-9-191-28781,

E-mail info-hecer@helsinki.fi, Internet www.hecer.fi 


\title{
A Naïve Sticky Information Model of Households' Inflation Expectations*
}

\begin{abstract}
This paper provides a simple epidemiology model where households, when forming their inflation expectations, rationally adopt the past release of inflation with certain probability rather than the forward-looking newspaper forecast as suggested in Carroll [2003, Macroeconomic Expectations of Households and Professional Forecasters, Quarterly Journal of Economics, 118, 269-298]. The posterior model probabilities based on the Michigan survey data strongly support the proposed model. We also extend the agentbased epidemiology model by deriving for it a simple adaptation, which is suitable for estimation. Our results show that this model is able to capture the heterogeneity in households' expectations very well.
\end{abstract}

JEL Classification: C11, C53, C82, D84, E31.

Keywords: Inflation expectations, heterogeneous expectations, survey expectations, sticky information, Bayesian analysis.

Markku Lanne

Department of Economics

University of Helsinki

P.O. Box 17 (Arkadiankatu 7)

FI-00014 University of Helsinki

FINLAND

e-mail: markku.lanne@helsinki.fi
Arto Luoma

Department of Mathematics and Statistics University of Tampere

FI-33014 University of Tampere

FINLAND

e-mail: arto.luoma@uta.fi

Jani Luoto

School of Business and Economics

University pf Jyväskylä

P.O. Box 35

40014 University of Jyväskylä

FINLAND

* Markku Lanne acknowledges financial support from the Academy of Finland. 


\section{Introduction}

In recent years there has been an increasing interest in explaining agents' inflation expectations formation process; see, e.g., Mankiw and Reis (2006, 2007), Sims (2006), Trabandt (2007) and Branch $(2004,2007)$. This is mainly due to observed failure of the rational expectation hypothesis. Within this literature, Mankiw and Reis (2002) propose a simple sticky information model where agents know the true probability distribution of the economy, but update their information set each period with certain probability. Carroll $(2003,2006)$ and Reis $(2006 a, b)$ seek microfoundations for sticky information models, while Mankiw et al. (2003) and Carroll (2003, 2006) find evidence based on survey data supporting these models; see also Khan and Zhu (2006), Andres et al. (2005), Kiley (2007), Coibion (2006, 2007), and Doepke et al. (2008). Finally, Branch (2007) bridges the sticky information and heterogeneous expectations literatures by presenting empirical evidence in favor of both, model heterogeneity and limited information flows; see Branch (2007) and references therein.

Closest to our work, Carroll (2003) develops and estimates an expectation formation model, where the general public adopt professionals' forecast with certain probability, rather than form their own rational forecasts. The structure of his model was inspired by simple models of disease spread from the epidemiology literature, and it provides promising microfoundations for sticky information models. To the best of our knowledge, it is also unique in relaxing the assumption that an ordinary person either knows the true probability distribution of the economy or can estimate some sophisticated econometric model ${ }^{1}$ when forming expectations. This relaxation is, however, important, since although trained economists might have this kind of knowledge, it would probably be an overwhelming task for an ordinary consumer (producer); see Shiller (1997). According to Carroll (2003), it might require, for example, obtaining a Ph.D. degree in economics first.

Despite the virtues of Carroll's (2003) model, recent work has cast doubt on the reliability of professionals' inflation forecasts, and, in general, on traditional approaches to inflation forecasting; see e.g. Atkeson and Ohanian (2001), Fisher et al. (2002), Sims (2002), Stock and Watson (2002), and Brave and Fisher (2004). In particular, Atkeson and Ohanian (2001) found that since 1984 the one-year-ahead inflation forecast of professionals ${ }^{2}$ has not been better than the "naïve" forecast given by the inflation rate over the previous year. Thus, it is natural to question the rationale of

\footnotetext{
${ }^{1}$ That is, agents are assumed to be 'boundedly' rational; see e.g. Evans and Honkapohja (2001).

${ }^{2}$ Specifically, Federal Reserve's Greenbook forecasts.
} 
searching for relatively rare newspaper forecasts (or to form one's own rational forecast), when the most recently reported past inflation statistic provides a competitive forecast 'model' for future inflation. In this paper, we propose an epidemiology model, where agents simply adopt with certain probability the past release of the annualized monthly inflation figure, the most commonly reported figure in the news coverage of inflation. We refer to this model as the naïve sticky information model, and test it empirically against Carroll's sticky information approach using quarterly U.S. data. Specifically, we compare posterior probabilities of the alternative models in which households update their expectations either to the forward-looking newspaper forecast or to the most recently reported past inflation statistic. As will be seen, U.S. data strongly support the latter.

Based on our empirical findings, we extend the agent-based epidemiology model, proposed by Carroll (2006), by deriving a relative simple adaptation of that model, suitable for estimation. The model assumes a constant personal probability for each agent to read a newspaper article on inflation. This variation in their newspaper reading propensities could explain differences in survey expectations across demographic groups, documented in Bryan and Venkatu (2001a, b) and Souleles (2004). The model differs from that of Carroll $(2003,2006)$ in that it no longer assumes agents to be 'infected' by rare newspaper forecasts. Rather, the source of 'infection' is the past release of annualized monthly inflation. The model is estimated with classified household-level survey data from 1981/3 to 2001/4 constructed by the Survey Research Center (SRC) at the University of Michigan. The results indicate that people on average update their expectations roughly once a year, which is in accordance with the previous literature, while their updating probabilities vary from 0.12 to 0.42 .

An agent-based epidemiology model also captures the overall heterogeneity between agents' expectations fairly well, in the sense that the variance of unexplained heterogeneity $\left(\sigma^{2}\right)$, i.e. heterogeneity in agents' expectations which the underlying model cannot explain, is quite small (approximately 1.1) relative to the high degree of heterogeneity observed in the actual micro level data. For example, in Branch's (2007) Rationally Heterogeneous Expectations (RHE) sticky information model, $\sigma^{2}$ was 36 . Although our result is not fully comparable to that of Branch (2007), we note that in the RHE model most variation in agents' expectations is attributed to unexplained heterogeneity ${ }^{3}$.

\footnotetext{
${ }^{3}$ The empirical standard deviation of Branch's (2007) sample was 12.7010. However, according to Branch (2007), the large empirical standard deviation is accounted for by a few outliers that expect inflation to be greater than $40 \%$. Since
} 
This paper is organized as follows. In Section 2, we discuss and estimate two alternative models where agents update their expectations either to the forward-looking newspaper forecast or to the most recently reported past inflation statistic. Section 3 provides an adaptation of the agent-based epidemiology model and estimates it using classified household-level survey data. Finally, Section 4 concludes the paper.

\section{Population Mean Analysis}

In this section, we introduce two alternative versions of an epidemiological expectation formation model and test them against each other. In both models agents face a constant probability of reading an article on inflation, and they believe that inflation follows a random walk; see Carroll (2003). However, in the first model, proposed by Carroll (2003), agents also believe that a forecast from a professional forecaster is more accurate than a forecast that they could construct themselves. In the alternative model, we give up this potentially invalid assumption.

\subsection{Naïve Sticky Information Model}

Epidemiological information structure provides promising microfoundations for sticky information models. In an epidemiology model, each individual at any time point faces a constant probability (say $\lambda$ ) of observing an article on inflation, which Carroll assumes to consist of professional forecasters' forecasts. Individuals who do not observe such an article simply continue to believe the last forecast they read about. This information structure leads to the following relationship between the mean inflation expectation of the general public $M_{t}[\cdot]$ and the newspaper forecast $N^{f}[\cdot]$ of professional forecasters at time $t$ (see Carroll, 2003, for a systematic treatment and references),

$$
M_{t}\left[\pi_{t+1}\right]=\lambda N_{t}^{f}\left[\pi_{t+1}\right]+(1-\lambda)\left\{\lambda N_{t-1}^{f}\left[\pi_{t+1}\right]+(1-\lambda)\left(\lambda N_{t-2}^{f}\left[\pi_{t+1}\right]+\ldots\right)\right\}
$$

his estimate of standard deviation of unexplained heterogeneity is 6 we state that in his model most variation in agents' expectations is attributed to unexplained heterogeneity. 
where $M_{t}[\cdot]$ is an operator that yields the population-mean value of the people's inflation expectations at time $t$ and $\pi_{t+1}$ is measured as the quarterly mean of monthly inflation of the seasonally adjusted Consumer Price Index (CPI) for all urban consumers. ${ }^{4}$

In what follows, model (1) can be written, using certain assumptions on the public's beliefs about the process of inflation, as

$$
\begin{aligned}
M_{t}\left[\pi_{t, t+4}\right] & =\lambda N_{t}^{f}\left[\pi_{t, t+4}\right]+(1-\lambda)\left\{\lambda N_{t-1}^{f}\left[\pi_{t-1, t+3}\right]+(1-\lambda)\left(\lambda N_{t-2}^{f}\left[\pi_{t-2, t+2}\right]+\ldots\right)\right\} \\
& =\lambda N_{t}^{f}\left[\pi_{t, t+4}\right]+(1-\lambda) M_{t-1}\left[\pi_{t-1, t+3}\right],
\end{aligned}
$$

where $\pi_{\tau, \tau+4}=\pi_{\tau+1}+\pi_{\tau+2}+\pi_{\tau+3}+\pi_{\tau+4}$ is the annual inflation from $\tau$ to $\tau+4$. Equation (2) provides a testable implication for the public's mean inflation expectations. It is based on two fundamental assumptions. Firstly, people believe that the economy has an underlying 'fundamental' inflation rate, $\pi_{t}^{f}$, and that the future changes in this fundamental rate are unforecastable. That is, they believe that $\pi_{t}=\pi_{t}^{f}+e_{t}$ and $\pi_{t+1}^{f}=\pi_{t}^{f}+\eta_{t+1}$, where $e_{t}$ is a transitory shock to the inflation rate, unforecastable beyond period $t$, while $\eta_{t+1}$ is a permanent innovation in the fundamental inflation rate, unforecastable beyond period $t+1$. Secondly, people also believe that professional forecasters have some deeper knowledge on how the economy works, and, therefore, are capable to estimate the past and present values of $e$ and $\eta$ through periods $t$ and $t+1$, respectively. These assumptions are in line with the near-unit-root behavior of the inflation rate, which is well documented in the empirical literature, and with the result of Shiller (1997) that ordinary persons do not know the causes of inflation. However, recent work has cast doubt on the reliability of professionals' inflation forecasts and the reliability of traditional approaches to forecasting inflation, suggesting that it might be rational for agents to use frequently reported actual inflation figures, rather than rare newspaper forecasts, as their inflation expectations; see e.g. Atkeson and Ohanian (2001), Fisher et al. (2002), Sims (2002), Stock and Watson (2002), and Brave and Fisher (2004).

\footnotetext{
${ }^{4}$ In this paper we use the quarterly series, since the only relevant candidate series for the views of professional forecasters which has the same forecasting horizon as the Michigan survey of households is the four-quarter inflation forecast from the Survey of Professional Forecasters (SPF). Furthermore, we use the quarterly means of monthly inflation series rather than quarterly inflation of the quarterly means of the monthly CPI series because a twelve-month forecast for annual inflation is the series that is asked from the respondents of the University of Michigan's Survey Research Center.
} 
To this end, we propose an alternative version of an epidemiological expectation formation model, which we call a naïve sticky information model. In the model, we follow Carroll and assume that people believe inflation to follow a random walk.

Equation (1) is based on the assumptions that every inflation article contains a complete forecast of the inflation rate for all future periods and that the agent who reads an article can recall the entire forecast. However, when these newspaper forecasts are on average no better than the naïve forecasts, people would eventually note this and rationally expect that $F_{t-j}\left[\pi_{t+1}\right]=N_{t-j}\left[\pi_{t-j}\right]$ $=N_{t-j}^{f}\left[\pi_{t+1}\right]$, where $j=0,1,2, \ldots, N_{t}\left[\pi_{t}\right]$ is the actual inflation reported in the news media $(N[\cdot])$ at time $t$, and $F$ is a forecast operator. Furthermore, no newspaper article contains an inflation forecast into infinite future. Rather, newspaper forecasts are rare compared to the frequently presented actual inflation figures. That is, the most recently reported inflation statistic $N_{t}\left[\pi_{t}\right]$ is the figure which people most probably observe when being influenced by the news media. Under these assumptions Equation (1) can be rewritten as

$$
\begin{aligned}
M_{t}\left[\pi_{t+1}\right] & =\lambda F_{t}\left[\pi_{t+1}\right]+(1-\lambda)\left\{\lambda F_{t-1}\left[\pi_{t+1}\right]+(1-\lambda)\left(\lambda F_{t-2}\left[\pi_{t+1}\right]+\ldots\right)\right\} \\
& =\lambda N_{t}\left[\pi_{t}\right]+(1-\lambda)\left\{\lambda N_{t-1}\left[\pi_{t-1}\right]+(1-\lambda)\left(\lambda N_{t-2}\left[\pi_{t-2}\right]+\ldots\right)\right\}
\end{aligned}
$$

Unfortunately, Equation (3) can be tested empirically only with annual data, since the available survey data only provide households' inflation expectations over the next year (i.e. their forecasts of annual inflation). Given the available dataset, we have roughly 20 annual observations, which are too few for valid inference. In addition, we prefer using quarterly data to keep the results comparable to those in Carroll $(2003,2006)$.

To derive a testable implication from Equation (3), let us note that under the random walk assumption, $\pi_{t, t+4}=\pi_{t, t+1}+v_{t+4}$, where $\pi_{t, t+1} \equiv 4 \pi_{t+1}$ is annualized inflation and $v_{t+4}$ a zero mean i.i.d. shock. Applying a lagged forecast operator on both sizes of this equation yields $F_{t-j} \pi_{t, t+4}=F_{t-j} \pi_{t, t+1}$ for any $j(j=0,1,2, \ldots)$. This holds since people believe that future values of $v_{t}$ are unforecastable. Furthermore, according to the random walk assumption, people also believe that the best predictor for the $t+1$ period annualized inflation, $F_{t-j} \pi_{t, t+1}$, is $N_{t-j}\left[\pi_{t-j-1, t-j}\right]$. Thus, we have the following empirically testable equation for the population mean of inflation expectations,

$$
M_{t}\left[\pi_{t, t+4}\right]=\lambda F_{t}\left[\pi_{t, t+4}\right]+(1-\lambda)\left\{\lambda F_{t-1}\left[\pi_{t, t+4}\right]+(1-\lambda)\left(\lambda F_{t-2}\left[\pi_{t, t+4}\right]+\ldots\right)\right\}
$$




$$
\begin{aligned}
& =\lambda N_{t}\left\lfloor\pi_{t-1, t}\right\rfloor+(1-\lambda)\left\{\lambda N_{t-1}\left\lfloor\pi_{t-2, t-1}\right\rfloor+(1-\lambda)\left(\lambda N_{t-2}\left\lfloor\pi_{t-3, t-2}\right\rfloor+\ldots\right)\right\} \\
& =\lambda N_{t}\left\lfloor\pi_{t-1, t}\right\rfloor+(1-\lambda) M_{t-1}\left\lfloor\pi_{t-1, t+3}\right\rfloor
\end{aligned}
$$

where $N_{t}\left[\pi_{t-1, t}\right]$ is the annualized inflation reported in the news media at time $t$. Equation (4) predicts the mean inflation expectation of the next year as a weighted average of the latest annualized inflation figure and the lagged mean inflation expectation. In our paper, annualized inflation is measured as (a quarterly mean of) annualized monthly inflation, the figure which the ordinary person most probably observes when being influenced by the news media.

\subsection{Econometric Approach}

Since the models in Equations (2) and (4) are non-nested, conventional tests cannot be used to the test them against each other. We therefore apply posterior model probabilities to explore if either of the models (2) or (4) is the true data generating process of the public's inflation expectation formation.

Given the data $y$, and competing models $M_{1}, \ldots, M_{K}$ with parameter vectors $\theta_{k}, k=1, \ldots, K$, the posterior model probability for model $M_{k}$ is given by

$$
p\left(M_{k} \mid y\right)=\frac{p\left(y \mid M_{k}\right) p\left(M_{k}\right)}{\sum_{i=1}^{K} p\left(y \mid M_{i}\right) p\left(M_{i}\right)}
$$

where

$$
p\left(y \mid M_{k}\right)=\int p\left(y \mid \theta_{k}, M_{k}\right) p\left(\theta_{k} \mid M_{k}\right) d \theta_{k},
$$

is the marginal likelihood of model $k, p\left(\theta_{k} \mid M_{k}\right)$ the prior density of $\theta_{k}$ under model $M_{k}, p\left(y \mid \theta_{k}, M_{k}\right)$ the likelihood, and $p\left(M_{k}\right)$ the prior probability of $M_{k}$. The explored models are standard linear regression models corresponding to theoretical models such as specifications (2) and (4). We will assume uniform independent prior distributions on given intervals for the regression coefficients. For simplicity, we also assume that the error variance $\sigma$ is, a priori, distributed uniformly on the interval $[0,2]$. Furthermore, we give the models equal prior probabilities, that is, $p\left(M_{k}\right)=1 / K, k=$ 
$1, . ., K$. Given these priors it is straightforward to derive analytical solutions for Equations (5) and (6).

However, since these uniform priors are not necessarily non-informative, we control our results using approximate posterior model probabilities based on the Schwarz Bayesian Information Criterion (BIC); see Schwarz (1978) and Garratt et al. (2007). Specifically,

$$
\ln p\left(y \mid M_{k}\right) \approx \ln p_{B I C}\left(y \mid M_{k}\right) \equiv l-\frac{\ln (T) \times p}{2}
$$

where $l$ denotes the log of the likelihood function evaluated at the maximum likelihood estimates, $p$ denotes the number of parameters in the model, and $T$ is the sample size. We use the previous marginal likelihood approximation, since it selects the same model as BIC, familiar to nonBayesians.

\subsection{Posterior Model probabilities}

We estimate Equations (2) and (4) using a monthly survey of inflation expectations of approximately 500 households, conducted by the Survey Research Center (SRC) at the University of Michigan, and the mean four-quarter inflation forecast from the Survey of Professional Forecasters $^{5}$ (SPF) as proxies for households' expectations and the newspaper forecast, respectively. The quarterly average series of annualized monthly inflation is based on seasonally adjusted real-time CPI (for all urban consumers) data ${ }^{6}$. The sample period ranges from 1981/3 to 2001/4, for which all the series are available. Specifically, we run the following regressions,

$$
\begin{aligned}
& M_{t}\left\lfloor\pi_{t, t+4}\right\rfloor=\alpha_{1} N_{t}\left\lfloor\pi_{t-1, t}\right\rfloor+\alpha_{2} M_{t-1}\left\lfloor\pi_{t-1, t+3}\right\rfloor+\varepsilon_{1, t}, \\
& M_{t}\left[\pi_{t, t+4}\right]=\alpha_{3} N_{t}^{f}\left[\pi_{t, t+4}\right]+\alpha_{4} M_{t-1}\left[\pi_{t-1, t+3}\right]+\varepsilon_{2, t},
\end{aligned}
$$

where $\varepsilon_{1, t}$ and $\varepsilon_{2, t}$ are assumed to be zero mean normally distributed errors with variances $\sigma_{1}{ }^{2}$ and $\sigma_{2}{ }^{2}$, respectively. We refer to Equations (8) and (9) as models $M_{1}$ (naïve model) and $M_{2}$ (Carroll's

\footnotetext{
${ }^{5}$ Data set is compiled by the Federal Reserve Bank of Philadelphia and is available at http://www.phil.frb.org/econ

${ }^{6}$ Data set is available at http://econweb.rutgers.edu/nswanson/realtime.htm
} 
model), respectively. In these models $\alpha_{1}+\alpha_{2}$ and $\alpha_{3}+\alpha_{4}$ are set at unity, since we are interested in situations where the epidemiology model can be treated as a structural description of the true process of the public's inflation expectations formation. We assume for the $\alpha_{1}$ and $\alpha_{3}$ uniform independent prior distributions on the interval $[0,1]$.

The upper panel of Table 1 gives the summary statistics for the models $M_{1}$ and $M_{2}$. According to the posterior model probabilities, there is a strong support in the data for model $M_{1}$. Specifically, the posterior probability of the naïve sticky information model being the true model exceeds 99.9 percent. Furthermore, the point estimate of $\alpha_{1}(0.18)$ is of sensible magnitude and in accordance with earlier empirical results. Conversely, the data do not lend support to model $M_{2}$; its posterior probability of being the true model is very close to zero.

Table 1. Posterior Model Probabilities for Mean Models

Estimated Models

\begin{tabular}{ccccccc}
$M_{t}\left[\pi_{t, t+4}\right]=\alpha_{1} N_{t}\left[\pi_{t-1, t}\right]+\alpha_{2} M_{t-1}\left[\pi_{t-1, t+3}\right]+\varepsilon_{1 t}$, and $M_{t}\left[\pi_{t, t+4}\right]=\alpha_{3} N_{t}^{f}\left[\pi_{t, t+4}\right]+\alpha_{4} M_{t-1}\left[\pi_{t-1, t+3}\right]+\varepsilon_{2 t}$ \\
\hline \multicolumn{7}{c}{ Estimates and Posterior Model Probabilities } \\
Models & $\alpha_{1}$ & $\alpha_{2}$ & $\alpha_{3}$ & $\alpha_{4}$ & $p\left(M_{k} \mid y\right)$ & $p_{B I C}\left(M_{k} \mid y\right)$ \\
$M_{1}$ & 0.18 & 0.82 & - & - & $99.993 \%$ & $99.997 \%$ \\
$M_{2}$ & $(0.02)$ & $(0.02)$ & - & 0.65 & $0.006 \%$ & $0.002 \%$
\end{tabular}

Estimated Model

$M_{t}\left[\pi_{t, t+4}\right]=\alpha_{5} N_{t}^{f}\left[\pi_{t, t+4}\right]+\alpha_{6} M_{t-1}\left[\pi_{t-1, t+3}\right]+\alpha_{7} N_{t}\left[\pi_{t-4, t}\right]+\varepsilon_{3 t}$

\begin{tabular}{cccccc} 
& \multicolumn{5}{c}{ Estimates and Posterior Model probabilities } \\
\cline { 3 - 5 } Models & $\alpha_{5}$ & $\alpha_{6}$ & $\alpha_{7}$ & $p\left(M_{k} \mid y\right)$ & $p_{B I C}\left(M_{k} \mid y\right)$ \\
$M_{3}$ & 0.45 & 0.64 & -0.09 & $0.001 \%$ & $0.001 \%$ \\
& $(0.09)$ & $(0.07)$ & $(0.07)$ & & \\
$M_{4}$ & 0.54 & 0.54 & -0.05 & $0.000 \%$ & $0.000 \%$ \\
& $(0.11)$ & $(0.09)$ & $(0.07)$ & & \\
\hline
\end{tabular}

All Equations are estimated over the period 1981:3 to 2001:4 for which real-time inflation, Michigan and SPF expectations series are available. Posterior standard errors (in parenthesis). There is no evidence of serial correlation or heteroscedasticity in the residuals of any reported regression.

Carroll (2003) suggests expanding model (9) to include the recently published annual inflation to test for the possibility that (a fraction of) individuals form their own forecast, the 'adaptive expectations' model, rather than use the forward-looking newspaper forecast. Using real-time CPI data, we therefore run the following regression

$$
M_{t}\left[\pi_{t, t+4}\right]=\alpha_{5} N_{t}^{f}\left[\pi_{t, t+4}\right]+\alpha_{6} M_{t-1}\left[\pi_{t-1, t+3}\right]+\alpha_{7} N_{t}\left[\pi_{t-4, t}\right]+\varepsilon_{3 t},
$$


where $N_{t}\left[\pi_{t-4, t}\right]$ is the annual inflation reported in the news media at time $t$. We estimate this model with and without the constraint $\alpha_{5}+\alpha_{6}+\alpha_{7}=1$ and refer to these models as $M_{3}$ and $M_{4}$, respectively. We assume for the $\alpha_{5}$ and $\alpha_{6}$ uniform independent prior distributions on the interval $[0,1]$ and for the $\alpha_{7}$ on $[-1,1]$. The results presented in the lower panel of Table 1 provide strong evidence against the adaptive expectations model. The posterior probabilities of the models $M_{3}$ and $M_{4}$ are virtually zero. Furthermore, the estimated coefficients of annual inflation are negative, which is counterintuitive, but their posterior intervals include zero. Carroll (2003), in contrast, obtained negative and statistically significant estimates. The fact that our results deviate from his probably follows from the differences in the data: we used real-time inflation figures, while he uses final vintage data. Finally, including a constant in the previous regressions does not alter the qualitative results. We will not present such results here, since, according to Carroll (2003), the presence of a positive constant term could reflect the effect of social transmission of inflation expectations (e.g. conversations with neighbors), in addition to the news-media channel explored in our paper.

In sum, these results are quite impressive in supporting the naïve sticky information model. However, there is likely to be heterogeneity in households' expectations that cannot be captured by the model. We will therefore, in the next section, estimate an agent-based version of the naïve sticky information model, where each individual has his own constant newspaper-reading propensity $\lambda$ and a fraction of population are allowed to round their inflation forecasts to the closest $0 \%, 5 \%, 10 \%$ or $15 \%$.

\section{An Agent-Based Epidemiology Model}

In this section we provide a relatively simple adaptation of an agent-based epidemiology model. We estimate it using classified household-level survey data of approximately 500 households, constructed by the Survey Research Center (SRC) at the University of Michigan. We use a quarterly dataset from 1981/3 to 2001/4 to keep results comparable to those presented in the previous section and in Carroll (2003, 2006). Furthermore, this dataset is easily available for the public. In it, the inflation expectation of each household is classified into one of seven categories. 


\subsection{The Model}

Inspired by the preliminary results presented in the previous section, we estimate an agent-based version of the epidemiological model, where, in any given period $t$, each agent faces a constant personal probability $\lambda$ of reading a newspaper article on the latest inflation figure. If he does not encounter such an article, his probability to use the views of period $t-1$ about inflation is $(1-\lambda) \lambda$. Generally, the probability that he uses the newspaper view of period $t-j+1$ in period $t$ is

$$
p(j \mid \lambda)=\lambda(1-\lambda)^{j-1}, \quad j=1,2, \ldots
$$

This is the probability function of the geometric distribution and we can easily compute an individual's $j$ with given $\lambda$. In the population level we need to choose a proper probability density function (p.d.f.) for $\lambda$. The beta distribution is a natural candidate for this purpose, since it is very flexible and assumes various shapes with different parameter values of $\alpha$ and $\beta$. Thus, we assume that across agents the p.d.f of $\lambda$ is

$$
p(\lambda)=\frac{1}{B(\alpha, \beta)} \lambda^{\alpha-1}(1-\lambda)^{\beta-1}, \quad \alpha>0, \beta>0
$$

where $B(\alpha, \beta)=\Gamma(\alpha) \Gamma(\beta) / \Gamma(\alpha+\beta)$ is the beta function and $\Gamma($.$) is the gamma function. The$ density (12) indicates that the higher the values of $\alpha$ and $\beta$, the more homogenous are agents' expectations. Combining functions (11) and (12) yields the following joint density function for $j$ and $\lambda$ :

$$
p(\lambda, j)=\frac{1}{B(\alpha, \beta)} \lambda^{\alpha}(1-\lambda)^{\beta+j-2}
$$

Integrating over all agents' updating probabilities $\lambda$, in turn, yields the following marginal probability function for $j$,

$$
p(j)=\frac{1}{B(\alpha, \beta)} \int_{0}^{1} \lambda^{\alpha}(1-\lambda)^{\beta+j-2} d \lambda=\frac{1}{B(\alpha, \beta)} B(\alpha+1, \beta+j-1)
$$




$$
=\frac{\alpha \Gamma(\alpha+\beta) \Gamma(\beta+j-1)}{\Gamma(\beta) \Gamma(\alpha+\beta+j)}, \quad j=1,2, \ldots
$$

Thus, $p(j)$ gives the probability of a randomly picked agent using the newspaper inflation view of period $t-j+1$ when forming his expectation at time $t$, assuming that $\lambda$ follows the beta distribution. Note that Eq. (14) is not in the form of any standard probability mass function. However, we can use numerical methods to estimate the mean, $E(\lambda)=\mu_{\lambda}$, and the variance, $\operatorname{Var}(\lambda)=\sigma_{\lambda}^{2}$, of $\lambda$, using the properties of the beta distribution ${ }^{7}$.

To complete the model we assume that each survey respondent reports

$$
\pi_{t, t+4}^{e}=N_{t-j+1}\left[\pi_{t-j, t-j+1}\right]+\varepsilon_{t+4}
$$

where his $j$ takes values from 1 to $K$ (we have truncated the distribution in Equation 14 such that $K$ $=28$ ), and $\varepsilon_{t+4}$ is a normally distributed error term with mean zero and variance $\sigma^{2}$. In Eq. (15) we assume that, after observing the newspaper article on inflation, an individual makes adjustments to the data and reports the figure that corresponds to his perception of future inflation. This is the standard modeling approach in the RHE literature where the stochastic terms $\varepsilon_{t}$ are interpreted as individual idiosyncratic shocks representing unobserved heterogeneity, i.e. heterogeneity in individuals' expectations which underlying model can not explain (see Branch, 2007, for discussion and references therein).

Given Equations (14) and (15) and the classified household-level survey data, the likelihood function for the sample of $T \times C$ observations, $n=\left(n_{11}, n_{12}, \ldots, n_{T C}\right)^{\prime}$, can be written as

$$
L(\theta ; n)=\prod_{t=l}^{T} \prod_{c=1}^{C}\left[P_{t}(c ; \theta)\right]^{n_{t c}}
$$

where $\theta=\left(\mu_{\lambda}, \sigma_{\lambda}, q, \sigma\right)^{\prime}$ is the vector of parameters, $n_{t c}$ the number of individuals in class $c$ at time $t$, and $P_{t}(c ; \theta)$ the probability that an individual belong the class $c$ at time $t$. The classes of individuals'

\footnotetext{
${ }^{7}$ The parameters $\alpha$ and $\beta$ can be solved as functions of $E(\lambda)$ and $\operatorname{Var}(\lambda)$ as follows: $\alpha=\mu_{\lambda}{ }^{2}\left(1-\mu_{\lambda}\right) / \sigma_{\lambda}{ }^{2}-\mu_{\lambda}$ and $\beta=\alpha\left(1 / \mu_{\lambda}-1\right)$.
} 
inflation expectations, $c=1, \ldots, 7$, are defined as follows: $1=-0 \%, 2=1-2 \%, 3=3-4 \%, 4=5 \%, 5$ $=6-9 \%, 6=10-14 \%, 7=15 \%+$. The probabilities $P_{t}(c ; \theta)$ are given by

$$
\begin{aligned}
& P_{t}(c=1 ; \theta)=(1-q)\left[\sum_{k=1}^{K} p(k) \int_{-\infty}^{0.5} p\left(\pi_{t, t+4}^{e} \mid j=k\right) d\left(\pi_{t, t+4}^{e}\right)\right]+q\left[\sum_{k=1}^{K} p(k) \int_{-\infty}^{2.5} p\left(\pi_{t, t+4}^{e} \mid j=k\right) d\left(\pi_{t, t+4}^{e}\right)\right] \\
& P_{t}(c=2 ; \theta)=(1-q)\left[\sum_{k=1}^{K} p(k) \int_{0.5}^{2.5} p\left(\pi_{t, t+4}^{e} \mid j=k\right) d\left(\pi_{t, t+4}^{e}\right)\right] \\
& P_{t}(c=3 ; \theta)=(1-q)\left[\sum_{k=1}^{K} p(k) \int_{2.5}^{4.5} p\left(\pi_{t, t+4}^{e} \mid j=k\right) d\left(\pi_{t, t+4}^{e}\right)\right] \\
& P_{t}(c=4 ; \theta)=(1-q)\left[\sum_{k=k}^{K} p(k) \int_{4.5}^{5.5} p\left(\pi_{t, t+4}^{e} \mid j=k\right) d\left(\pi_{t, t+4}^{e}\right)\right]+q\left[\sum_{k=1}^{K} p(k) \int_{2.5}^{7.5} p\left(\pi_{t, t+4}^{e} \mid j=k\right) d\left(\pi_{t, t+4}^{e}\right)\right] \\
& P_{t}(c=5 ; \theta)=(1-q)\left[\sum_{k=1}^{K} p(k) \int_{5.5}^{9.5} p\left(\pi_{t, t+4}^{e} \mid j=k\right) d\left(\pi_{t, t+4}^{e}\right)\right] \\
& P_{t}(c=6 ; \theta)=(1-q)\left[\sum_{k=1}^{K} p(k) \int_{9.5}^{14.5} p\left(\pi_{t, t+4}^{e} \mid j=k\right) d\left(\pi_{t, t+4}^{e}\right)\right]+q\left[\sum_{k=1}^{K} p(k) \int_{7.5}^{12.5} p\left(\pi_{t, t+4}^{e} \mid j=k\right) d\left(\pi_{t, t+4}^{e}\right)\right] \\
& P_{t}(c=7 ; \theta)=(1-q)\left[\sum_{k=1}^{K} p(k) \int_{14.5}^{\infty} p\left(\pi_{t, t+4}^{e} \mid j=k\right) d\left(\pi_{t, t+4}^{e}\right)\right]+q\left[\sum_{k=1}^{K} p(k) \int_{12.5}^{\infty} p\left(\pi_{t, t+4}^{e} \mid j=k\right) d\left(\pi_{t, t+4}^{e}\right)\right]
\end{aligned}
$$

where

$$
p\left(\pi_{t, t+4}^{e} \mid j=k\right)=\frac{1}{\sqrt{2 \pi} \sigma} \exp \left\{-\frac{1}{2}\left(\frac{\pi_{t, t+4}^{e}-N_{t-j+1}\left[\pi_{t-j, t-j+1}\right]}{\sigma}\right)^{2}\right\} .
$$

As we can see, the probability $P_{t}(c ; \theta)$ is calculated by integrating the conditional probability density $p\left(\pi_{t, t+4}^{e} \mid j=k\right)$ over the interval of class $c$ and summing over the possible updating intervals $k$ $=1, \ldots, K$. After observing actual household level survey data, we have also allowed for the possibility that a fraction $q$ of the total population round their inflation expectations to the closest $0 \%, 5 \%, 10 \%$, or $15 \%$. This kind of behavior may be typical of those agents who have no special interest in the economy (see, e.g., Bryan and Palmqvist, 2004). Note that the extreme of $q=0$ corresponds to the situation where there is no such behavior in the population. 


\subsection{Results}

We will estimate the previous model using Bayesian methods. The starting point of the Bayesian analysis is to determine the prior density function of the parameters, $p(\theta)$, which together with the likelihood function (16) yields the posterior density

$$
q(\theta \mid n)=\frac{p(\theta) L(\theta ; n)}{\int p(\theta) L(\theta ; n) d \theta}
$$

The prior density reflects the researcher's prior beliefs concerning plausible parameter values. Table 2 lists the marginal prior distributions of the parameters. A standard assumption on prior independence is used; see e.g. Zellner (1971). Reported marginal priors reflect the following parameter constraints, $0<\mu_{\lambda}<1,0<q<1,0<\sigma_{\lambda}$, and $0<\sigma$. The prior mean of $\mu_{\lambda}$ is set at 0.25 , which is a common result in previous studies. The prior mean of $\sigma_{\lambda}$ is set at 0.06 , indicating moderate variation in individuals updating probabilities; see Carroll (2006). We have not a clear idea about the value of $q$, hence the uniform prior $(\operatorname{Beta}(1,1))$ is entitled in this case. Furthermore, the prior mean of $\sigma$ is set at 5, slightly below the estimate of Branch (2007). Finally, with given prior variances, these marginal prior distributions turned out to be practically noninformative.

The estimation results of model (17) are reported in Table $2 .^{8}$ As can be seen, the median of the updating probability, $\mu_{\lambda}=0.38$, is higher than the point estimates of $\lambda$ reported in Table 1 , but it is in accordance with previous studies. The truncation of Equation (14) is most likely the reason for the difference between the results. For comparison, we also estimated a truncated version of Equation (4) with $K=28,{ }^{9}$ based on Michigan mean expectations and obtained an estimate of $\lambda$ quite close to that obtained for model (17). ${ }^{10}$

\footnotetext{
${ }^{8}$ We used the Metropolis algorithm to generate a Monte Carlo sample from the posteriors. The algorithm uses the multivariate normal distribution for the jump distribution on changes in the parameters $\theta$. The inverse of the Hessian of the $\log$ posterior density at the posterior mode, scaled by the factor $2.4^{2} / 4\left(2.4^{2} / 5\right)$, is used to obtain an optimal covariance matrix of the multivariate normal jump distribution; see e.g. Gelman et al. (2004). We use 10,000 draws, discarding the first 2,000 as a burn-in period. As a convergence check, three chains with different randomly selected starting values are simulated. The potential scale reduction factor of Gelman and Rubin (1992) was between 1 and 1.04 for each parameter. The multivariate version of Gelman and Rubin's diagnostic, proposed by Brooks and Gelman (1997), were between $1.00-1.01$ for each model. Finally, the frequency of accepted jumps was roughly 0.28 .

${ }^{9}$ The results of the regressions with shorter and longer lag lengths are quite similar.

${ }^{10}$ We also estimated model (17) with the SPF series and found the estimate of the updating probability to be very high $(\approx 1)$. The probabilities $\lambda$ were also very homogenous ( $\sigma_{\lambda}$ was close to zero). When estimating a truncated version of Equation (2) based on Michigan and SPF mean expectations, an estimate of $\lambda$ close to 1 was also obtained. These implausibly high estimates lend support to the naïve sticky information model.
} 
The median of the standard deviation of $\lambda$ is estimated to be 0.12 , indicating strong heterogeneity in agents' newspaper reading habits. The updating probability $\lambda$ varies from 0.05 to 0.85 among agents according to our simulation experiment (not reported here) ${ }^{11}$. This strong variation in their newspaper reading propensities could explain the differences in survey expectations across demographic groups, documented in Bryan and Venkatu (2001a, b) and Souleles (2004). Furthermore, roughly $1 / 5(q=0.22)$ of the population round their inflation forecasts to the closest $0 \%, 5 \%, 10 \%$ or $15 \%$. This result is consistent with the shape of the empirical distribution of individual level data. The standard deviation of idiosyncratic shocks, $\sigma$, is estimated to be 4.3 .

Table 2. Priors and Posteriors for Agent-Based Epidemiology Models

The Likelihood

$$
L(\theta ; n)=\prod_{t=l}^{T} \prod_{c=1}^{C}\left[P_{t}(c ; \theta)\right]^{n_{t c}}
$$

\begin{tabular}{|c|c|c|c|c|c|c|c|}
\hline & \multicolumn{3}{|c|}{ Prior Distributions } & \multicolumn{2}{|c|}{$\begin{array}{c}\text { Posterior Distr. } \\
\text { Model (17) }\end{array}$} & \multicolumn{2}{|c|}{$\begin{array}{c}\text { Posterior Distr. } \\
\text { Model (19) }\end{array}$} \\
\hline & Distr. & Mean & St.Dev. & Median & St.Dev. & Median & St.Dev. \\
\hline & \multicolumn{7}{|c|}{$1981 / 3-2001 / 4$} \\
\hline$\mu_{\lambda}$ & Beta & 0.25 & 0.38 & 0.379 & 0.006 & 0.378 & 0.005 \\
\hline$\sigma_{\lambda}$ & Invgamma & 0.06 & 0.19 & 0.116 & 0.004 & 0.099 & 0.004 \\
\hline$\sigma$ & Invgamma & 5.00 & 11.00 & 4.337 & 0.014 & 2.171 & 0.027 \\
\hline$q$ & Beta & 0.50 & 0.38 & 0.217 & 0.002 & 0.207 & 0.002 \\
\hline$a$ & Gamma & 1.00 & 1.00 & - & - & 0.548 & 0.012 \\
\hline \multirow{3}{*}{$\begin{array}{c}\sigma_{0} \\
p\left(M_{k} \mid y\right)\end{array}$} & - & - & - & - & - & 1.607 & 0.017 \\
\hline & & & & \multicolumn{2}{|c|}{0.000} & \multicolumn{2}{|c|}{1.000} \\
\hline & \multicolumn{7}{|c|}{$1984 / 1-2001 / 4$} \\
\hline$\mu_{\lambda}$ & Beta & 0.25 & 0.38 & 0.193 & 0.002 & 0.220 & 0.005 \\
\hline$\sigma_{\lambda}$ & Invgamma & 0.06 & 0.19 & 0.008 & 0.002 & 0.024 & 0.007 \\
\hline$\sigma$ & Invgamma & 5.00 & 11.00 & 3.876 & 0.015 & 1.295 & 0.020 \\
\hline$q$ & Beta & 0.50 & 0.38 & 0.208 & 0.002 & 0.204 & 0.002 \\
\hline$a$ & Gamma & 1.00 & 1.00 & - & - & 0.669 & 0.019 \\
\hline$\sigma_{0}$ & - & - & - & - & - & 1.061 & 0.021 \\
\hline$p\left(M_{k} \mid y\right)$ & & & & \multicolumn{2}{|c|}{0.000} & \multicolumn{2}{|c|}{1.000} \\
\hline
\end{tabular}

We set $K$ at 28 (i.e. seven years) to have a sufficiently good approximation for Equation (14). The results of the regressions with shorter and longer lag lengths are quite similar. The inflation data before 1978:2 were based on the quarterly means of monthly inflation of the lagged CPI series (lagged by one month), which was acquired from Norman R. Swanson's home page.

According to specification (17), a typical person is able to remember past inflation figures correctly. We are, however, rather skeptical about this, as it seem more likely that individuals' ability to

\footnotetext{
${ }^{11}$ Given $\alpha$ and $\beta$ the updating frequency $\lambda$ can be simulated from the beta distribution (12) and given updating frequency $(\lambda) j$ can be simulated from the geometric distribution (11).
} 
remember the contents of news articles decreases over time. To allow for this, we assume a very simple linear model for the error variance, related to recalling the newspaper figure. Specifically, the variance in (17) is parametrized as $\sigma^{2}(j)=\sigma^{2}(3(j-1)+a)$ and, thus, increases by $\sigma^{2}$ every month. Furthermore, we can interpret $\sigma_{0}^{2} \equiv \sigma^{2} a$ as the variance in individuals' expectations not explained by the memory weakening process. With time-varying variance, model (17) can be written in the form

$$
p\left(\pi_{t, t+4}^{e} \mid j=k\right)=\frac{1}{\sqrt{2 \pi} \sigma(j)} \exp \left\{-\frac{1}{2}\left(\frac{\pi_{t, t+4}^{e}-N_{t-j+1}\left[\pi_{t-j, t-j+1}\right]}{\sigma(j)}\right)^{2}\right\} .
$$

The results in Table 2 lend strong support to the specification (19). In particular, the probability of the time-varying variance model being the true model is virtually one. ${ }^{12}$ Furthermore, the results concerning the median value of $\lambda$ and the heterogeneity of $\lambda$ between agents are close to those obtained for model (17). This model can explain agents' overall heterogeneity fairly well, in the sense that the standard deviation of unexplained heterogeneity $\left(\sigma_{0} \approx 1.6\right)$ is small relative to the high degree of heterogeneity observed in the actual data. According to our simulation experiment (not reported) this model can easily explain the long tails observed in individual level data. In Branch's (2007) RHE sticky information model, unexplained heterogeneity explains these long tails. In particular, the empirical standard deviation of Branch's (2007) sample was 12.7010. However, according to Branch (2007), the large empirical standard deviation is accounted for by a few outliers with expected inflation to be greater than $40 \%$. Since his estimate for the standard deviation of unexplained heterogeneity was 6 , we state that most variation in agents' expectations is in his model attributed to unexplained heterogeneity.

Since the forecasts based on standard econometric methods have not on average been better than the naïve forecast since 1984 (see Atkeson and Ohanian, 2001), we may expect that a fraction of individuals have used newspaper forecasts before that date. This might disturb the previous results. Therefore, the results should be checked using a post 1984 sample. Table 2 shows the estimation results of a sample from 1984/1 to 2001/4. The median estimate of $\lambda$ is now close to the estimate of the corresponding parameter in the model of Section $2.4(0.18)$, which is quite good news for the

\footnotetext{
12 The marginal likelihoods are estimated from the simulated posterior samples using the reciprocal importance estimator (see Gelfand and Dey, 1994) with a truncated multivariate normal importance density proposed by Geweke (1999).
} 
naïve sticky information model. The heterogeneity of $\lambda$ between agents $\left(\sigma_{\lambda}=0.024\right)$, obtained for model (19), is lower than that obtained for the full sample, but markedly higher than that obtained for the constant variance model $\left(\sigma_{\lambda}=0.008\right)$. In this sample, the updating probability $\lambda$ varies from 0.12 to 0.42 among agents according to our simulation experiment (not reported). However, most of the probability mass of $\lambda$ lies in interval $[0.15,0.3]$, indicating a moderate degree of heterogeneity. This evidence is in accordance with the 'implicit' evidence of Carroll (2006). Finally, the standard deviation of unobserved (or unexplained) heterogeneity between individuals ( $\sigma_{0} \approx 1.1$ ) is close to that obtained for the full sample and indicates that an agent-based naïve sticky information model does a fairly good job in capturing the heterogeneity in individuals' expectations.

\section{Conclusion}

Mankiw and Reis (2002) have proposed sticky information as an alternative to the sticky prices of Calvo (1983). Carroll (2003) has provided microfoundations for the aggregate inflation expectations equation of Mankiw and Reis (2002). The model presented in this paper can be interpreted as an extension of Carroll's (2003) model. We have proposed that agents, when forming their inflation expectations, adopt the past release of annualized monthly inflation with certain probability rather than the forward-looking newspaper forecast as suggested in Carroll (2003). The model is motivated by recent empirical work, which has cast doubt on the reliability of professionals' inflation forecasts, and, in general, on traditional approaches to inflation forecasting. We have shown that this simple model is able to fit the inflation expectations data of the Michigan survey very well. In particular, the model can capture not only aggregate inflation expectations, but also the observed heterogeneity in households' expectations. The latter finding is based on a relatively simple adaptation, which we have derived in this study for Carroll's (2006) agent-based epidemiology model. 


\section{References}

Andres, J., Lopez-Salido, D., Nelson, E., 2005. Stick-Price Models and the Natural Rate Hypothesis. Journal of Monetary Economics 52, 1025-1053.

Atkeson, A., Ohanian, L.E., 2001. Are Phillips curves useful for forecasting inflation?. Quarterly Review, Federal Reserve Bank of Minneapolis 25, 2-11.

Branch, W.A., 2004. The theory of rationally heterogeneous expectations: evidence from survey data on inflation expectations. Economic Journal 114, 592-621.

Branch, W.A., 2007. Sticky information and model uncertainty in survey data on inflation expectations. Journal of Economic Dynamics and Control 31, 245-276.

Brave, S., Fisher, J.D., 2004. In search of a robust inflation forecast. Economic Perspectives 28, 1231.

Brooks, S.P., Gelman, A., 1997. General methods for monitoring convergence of iterative simulations. Journal of Computational and Graphical Statistics 7, 434 - 455.

Bryan, M., Palmqvist, S. 2004. Testing near-rationality using survey data. Sveriges Riksbank Working Paper No. 183.

Bryan, M., Venkatu, G., 2001a. The demographics of inflation opinion surveys. Economic Commentary, Federal Reserve Bank of Cleveland.

Bryan, M., Venkatu, G., 2001b. The curiously different inflation perspectives of men and women. Economic Commentary, Federal Reserve Bank of Cleveland.

Calvo, G., 1983. Staggered Prices in a Utility Maximizing Framework. Journal of Monetary Economics 12, 383-98.

Carroll, C.D., 2003. Macroeconomic Expectations of Households and Professional Forecasters. Quarterly Journal of Economics 118, 269-298.

Carroll, C.D., 2006. The Epidemiology of Macroeconomic Expectations. in The Economy as an Evolving Complex System, III, ed. by Larry Blume, and Steven Durlauf. Oxford University Press.

Coibion, O., 2006. Inflation Inertia in Sticky Information Models. Contributions In Macroeconomics 6, Iss. 1, Article 1.

Coibion, O., 2007. Testing the Sticky Information Phillips Curve. Manuscript.

Doepke, J., Dovern, J., Fritsche, U., Slacalek, J., 2008. The Dynamics of European Inflation Expectations. The B.E. Journal of Macroeconomics 8, Iss. 1 (Topics), Article 12.

Evans, G.W., Honkapohja, S., 2001. Learning and Expectations in Macroeconomics. Princeton University Press, Princeton, NJ. 
Fisher, J.D., Liu, C., Zhou, R., 2002. When can we forecast inflation?. Economic Perspectives, Federal Reserve Bank of Chicago, First Quarter, 30-42.

Garratt, A., Koop, G., Mise, E., Vahey, S.P., 2007. Real-time Prediction with UK Monetary Aggregates in the Presence of Model Uncertainty. Birkbeck Working Papers in Economics and Finance, No 714.

Gelfand, A., Dey, D., 1994. Bayesian model choice: Asymptotic and exact calculations. Journal of Royal Statistical Society Ser. B 56, $501-514$.

Gelman, A, Rubin, D.B., 1992. Inference from iterative simulation using multiple sequences. Statistical Science 7, $457-472$.

Gelman, A., Carlin, J.B., Stern, H.S., Rubin, D.B., 2004. Bayesian Data Analysis $2^{\text {nd }}$ edition, Chapman \& Hall/CRC.

Geweke, J., 1999. Using Simulation Methods for Bayesian Econometric Models:

Inference, Development and Communication. Econometric Reviews 18, 1-126.

Khan, H., Zhu, Z., 2006. Estimates of the Sticky-Information Phillips Curve for the United States. Journal of Money, Credit and Banking 38, 195-207.

Kiley, M.T., 2007. A Quantitative Comparison of Sticky-Price and Sticky-Information Models of Price Setting. Journal of Money, Credit and Banking 39, 101-125.

Mankiw, N.G., Reis R., 2002. Sticky Information Versus Sticky Prices: A Proposal to Replace the New Keynesian Phillips Curve. Quarterly Journal of Economics 117, 1295-1328.

Mankiw, N.G., Reis R., 2006. Pervasive Stickiness. American Economic Review Papers and Proceedings 96, 164-169.

Mankiw, N.G., Reis R., 2007. Sticky Information in General Equilibrium. Journal of the European Economic Association 5, 603-613.

Mankiw, N.G., Reis, R., Wolfers, J., 2003. Disagreement about inflation expectations. In: Gertler, M., Rogoff, K. (Eds.). NBER Macroeconomics Annual 2003.

Reis, R., 2006a. Inattentive Consumers. Journal of Monetary Economics 53, 1761-1800.

Reis, R., 2006b. Inattentive Producers. Review of Economic Studies 73, 793-821.

Schwarz, G., 1978. Estimating the dimension of a model. Annals of Statistics 6, 461-464.

Shiller, R.J., 1997. Why Do People Dislike Inflation. in Reducing Inflation: Motivation and Strategy, edited by Christina D. Romer and David H. Romer. Chicago: University of Chicago Press.

Stock, J.H., Watson, M., 2002. Macroeconomic forecasting using diffusion indexes. Journal of Business and Economic Statistics 20, 147-162. 
Sims, C., 2002. The role of models and probabilities in the monetary policy process. Brookings Papers on Economic Activity 2, 1-62.

Sims, C.A., 2006. Rational Inattention: A Research Agenda. Manuscript.

Souleles, N.S., 2004. Expectations, Heterogeneous Forecast Errors, And Consumption: Micro

Evidence Form The Michigan Consumer Sentiment Surveys. Journal of Money, Credit and Banking 36, 39-72.

Trabandt, M., 2007. Sticky Information vs. Sticky Prices: A Horse Race in a DSGE Framework. Kiel Working Papers No. 1369.

Zellner, A., 1971. An Introduction to Bayesian Inference in Econometrics. J. Wiley and Sons, Inc., New York. 